\title{
Sexsomnie: Schlafstörung mit gravierenden Folgen
}

\author{
Sexsomnie ist selten, kann aber Konsequenzen für \\ Betroffene und Partner haben, die weit über eine \\ nächtliche Belästigung hinausgehen.
}

Sexsomnie gehört wie Schlafwandeln (Somnambulismus) oder Nachtschreck (Pavor nocturnus) zu den Non-REM-Parasomnien. Sie ist eine atypische sexuelle Verhaltensstörung während des Schlafs: Betroffene masturbieren, entwickeln spontane Orgasmen, verwenden erotische Sprache („sleep sex talking“) oder versuchen beziehungsweise vollziehen Geschlechtsverkehr. Das alles ohne aus dem Non-REM-Schlaf zu Bewusstsein zu kommen und sich an die Handlungen erinnern zu können. Die Schlafstörung verläuft oft chronisch und rezidivierend. In seltenen Fällen empfinden Betroffene und Bettpartner die Folgen der Sexsomnie durchaus nicht als negativ, weil der Sex angenehmer, verrückter („kinky“) und weniger hastig sein kann, weil Betroffene auch sanfter, leidenschaftlicher und partnerorientierter agieren können als im Wachzustand, erklärte Professor Carlos H. Schenck, University of Minnesota Medical School Minneapolis (USA). Weitaus häufiger sind die Folgen jedoch negativ und reichen bis zu forensischen Konsequenzen. Bettpartner sind schockiert und verängstigt, das oft nicht einvernehmliche sexuelle Verhalten kann von „Begrabschen“ über aggressives Verhalten bis zur Vergewaltigung reichen. Bei Betroffenen stehen Scham und Schuldgefühle im Vordergrund, Verwirrung und Verzweiflung - nicht zuletzt aufgrund der Amnesie hinsichtlich der ihnen nicht bewussten Handlungen.

\section{Juristische Folgen sind häufig}

Die Daten eines Literaturreviews, die Schenck in Münster präsentierte, zeigen, dass Männer etwa dreimal häufiger betroffen sind als Frauen, das mittlere Alter liegt bei 35 Jahren, die Krankheitsdauer bei sieben Jahren [Schenck CH et al. Sleep 2007; 30 (6): 683 - 702]. $24 \%$ der Patienten masturbieren, $19 \%$ sprechen erotisch, je $37 \%$ berühren ihren Partner oder verhalten sich aggressiv, in $50 \%$ kommt es zum Geschlechtsverkehr. Auch ,sleepsex" mit Minderjährigen ist nicht ausgeschlossen (20\%). In einem Viertel der Fälle kommt es zu juristischen Folgen. Fast immer können sich Betroffene an ihre Handlungen jedoch nicht erinnern (96\%). Aktuelle Untersuchungen weisen auf Trigger der Sexsomnie wie zirkadiane Rhythmusstörungen (etwa durch Schichtarbeit), sexuelle Traumata in der Adoleszenz, familiäre Häufung sowie Parkinson und dopaminerge Therapie hin. Sexsomnie ist weder mit gesteigertem Sexualtrieb noch mit sexueller Deprivation oder Depravation assoziiert. Therapeutisch ist Clonazepam häufig erfolgreich (86\%). Patienten mit obstruktivem Schlafapnoesyndrom (OSAS) als Trigger der Störung profitieren immer von einer CPAP-Beatmung (continuous positive airway pressure); auch für MAD (mandibular advancement device) sind Erfolge beschrieben.

Michael Koczorek

Symposium "Sexolepsie oder Sexsomnie? Anfälle mit sexuellen Verhaltensschablonen im Rahmen von Parasomnie oder Epilepsie“; 25. Kongress der Deutschen Gesellschaft für Schlafforschung und Schlafmedizin, Münster, 9.11.2017

\section{Sexolepsie oder Sexsomnie? Die Differenzialdiagnose ist komplex}

Nicht nur Parasomnien, sondern auch epileptische Anfälle können komplexe sexuelle Verhaltensmuster verursachen. Das macht die Differenzialdiagnose nicht einfacher.

Als Symptom der Sexsomnie imponieren komplexe sexuelle Verhaltensschablonen im Schlaf. Einfache sexuelle Sensationen oder Verhaltensmuster sind dagegen im Rahmen epileptischer Anfälle beschrieben. Dazu gehören etwa unspezifische sensible Missempfindungen im Genital bei parietalen oder insulären Anfällen, angenehme erotische Wahrnehmungen bei Anfällen des non-dominanten Temporallappens und genitale Automatismen mit nicht zielgerichtetem Nesteln im Genitalbereich bei Temporallappenanfällen. Auch kurz dauernde, simple, gelegentlich zielgerichtet wirkende hypermotorische sexuelle Handlungsschablonen bei temporalen und vor allem frontalen Anfällen zählen dazu.

Eine retrospektive Fallsammlung zeige nun, dass auch epileptische Anfälle komplexe sexuelle Verhaltensschablonen (KSVS) verursachen können, berichtete Dr. Berthold Voges, Fachbereich Neurologie und Epileptologie am Evangelischen Krankenhaus Alsterdorf, Hamburg. Ausgewertet wurden die Daten von 4.600
Patienten, die zwischen 2013 und 2016 im Epilepsiezentrum Hamburg per Video-EEG-Monitoring untersucht worden waren. KSVS als Manifestation epileptischer Anfälle wurde bei vier Patientinnen beobachtet. Der Anfallsursprung war zweimal temporal, zweimal temporal und frontal. EEG, SPECT, MRT und Histopathologie (bei drei operierten Patientinnen) sowie postoperative Anfallsfreiheit bei zwei Patientinnen untermauerten Voges zufolge die Hypothese hinsichtlich der epileptogenen Zone. Ferner wurden zwei männliche Patienten mit KSVS als Manifestation von Non-REM-Parasomnien identifiziert. „In unserer Kohorte war KSVS also ein seltenes iktales Phänomen, das überwiegend auf non-dominanten temporalen oder frontalen Anfallsbeginn hinwies", so Voges.

Für die Differenzialdiagnose relevant sind der Beginn und die Dauer der Störung, ihre Semiologie und ihr Ablauf sowie Erinnerung und Einbettung ( $\bullet$ Tab. 1). Keines der beschriebenen Merkmale konnte allerdings eine der beiden Diagnosen sicher beweisen oder ausschließen: Interaktionsfähigkeit oder lang dauerndes und zielgerichtetes sexuelles Verhalten bis zum vollendeten Beischlaf wurden auch bei epileptisch bedingten KSVS gefunden. Epilepsie müsse laut Voges daher als mögliche Ursache für KSVS auch in Betracht gezogen werden. Zur sicheren Unterscheidung zwischen Epilepsie und Parasomnie kön- 